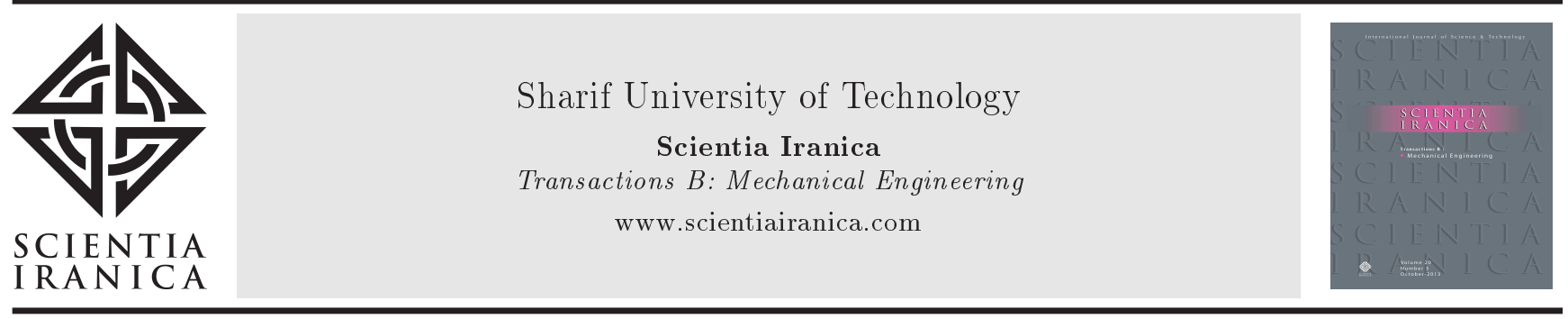

\title{
Unsteady impulsive oblique stagnation-point flow impinging axisymmetrically on a vertical circular cylinder with mixed convection heat transfer
}

\author{
R. Bayat and A.B. Rahimi* \\ Faculty of Engineering, Ferdowsi University of Mashhad, Mashhad, P.O. Box 91775-1111, Iran.
}

Received 21 July 2015; received in revised form 2 March 2016; accepted 20 June 2016

KEYWORDS
Unsteady; Oblique
stagnation-point flow;
Axisymmetric;
Vertical circular
cylinder;
Mixed convection.

\section{Introduction}

Due to various industrial applications of stagnationpoint flow to a plate or cylinder, many studies have been done on these issues. For example, in manufacturing of metal, plastic, or food product by extrusion process, the output product is usually cooled by blowing a peripheral fluid flow. Since the cooling process affects the resistance and quality of the product, analytical modeling and evaluation of this physical event are highly of interest under the topic of stagnation flow problem. By using analytical methods, particularly similarity solutions, accurate results can be obtained demonstrating flow behavior in a viscous boundary

\footnotetext{
*. Corresponding author. Tel.: +985138805018

E-mail address: rahimiab@um.ac.ir (A.B. Rahimi)
}

layer. However, finding the appropriate similarity variables and solving the governing differential equations of the problem are the main challenges of this approach. The history of analytical methods studies using similarity solution techniques goes back to Hiemenz [1]. He investigated the steady two-dimensional laminar incompressible flow perpendicularly impinging on a flat plate and succeeded to transform governing equations into an ordinary differential equation, which was then solved by using numerical methods. Wang [2] was the first who presented the solution of stagnationpoint flow on a circular cylinder. This problem was specific to a simple case of a stationary cylinder without suction and blowing in steady-state condition, and its importance was due to introduction of different similarity variables into cylindrical coordinate system. Based on Wang's accomplishment, Gorla [3] could 
obtain both velocity and temperature distributions for a cylinder with constant wall temperature and constant heat flux. He considered energy equation and used a transformation for temperature quantity, then solved the extracted differential equations by numerical methods. Gorla [4] continued his work and solved the problem for transient state, when the free stream flow has time-dependent velocity. $\mathrm{He}$ used series solutions in his analysis and presented the results of some specific time-dependent functions. Gorla [5], in his next paper, assumed the cylinder having harmonic motion and obtained the results of this unsteady problem for two cases of low and high frequencies. Takhar et al. [6] investigated the unsteady case of this problem for any arbitrary time-dependent free-stream or cylinder velocities. They obtained numerical methods for solving their final differential equations for the cases of semi-similar and self-similar forms. Stuart [7], for the first time, formulated the problem of oblique stagnation-point flow on a flat plate by involving vorticity in the impinging velocity field. The first formulation of the oblique stagnationpoint flow on a circular cylinder was accomplished by Okamoto [8]. He produced a stream function for the oblique stagnation-point flow by considering an additional term of shear flow along the cylinder's axis and solved the additional related equation, numerically. Another study on oblique stagnation-point flow on a circular cylinder was conducted by Wideman et al. [9] They solved the functions used in similarity solution by asymptotic analysis method for high Reynolds numbers. In their paper, Ramachandran et al. [10] dealt with solving the problem of normal stagnationpoint flow against a vertical flat plate in the presence of buoyancy forces which cause mixed convection. Gorla [11] studied mixed convection of a vertical circular cylinder impinged by a normal stagnationpoint flow and presented a numerical solution to the equations in the case of constant or linear variable wall temperature. Lok et al. [12] solved the problem of the oblique stagnation-point flow on a vertical flat plate along with mixed convection heat transfer with wall's constant heat flux condition to obtain the velocity field and the streamlines. Revnic et al. [13] dealt with mixed convection heat transfer problem of a circular cylinder impinged by a normal stagnation-point flow, already mentioned by Gorla [11] whose solution was presented for a wider range of dimensionless numbers.

In the present analysis, the unsteady problem of mixed convection heat transfer of a vertical circular cylinder impinged by an impulsive oblique stagnationpoint flow is dealt with; moreover, some characteristics are investigated in terms of the angle and time of flow obliqueness. This problem may be a simulation of certain practical cases where some inclined peripheral blowers around a long rod are employed for cooling purposes. By focusing on time interval at the beginning of the process, we encounter an unsteady situation which is caused by the sudden blowing fluid meeting a hot surface. In this problem, a constant-strength oblique axisymmetric outer flow impinges on a circular cylinder, impulsively. The flow is considered inviscid far from the cylinder, but in the region near the surface (boundary layer), equations of viscous flow are reduced to a system of coupled semi-similar ODEs by using similarity transformations and are solved numerically using a finite-difference scheme to determine velocity and temperature distributions of the flow filed. This solution is conducted for any arbitrary angle of outer flow in the range of zero to near $90^{\circ}$ for both conditions of assisting or opposing buoyancy forces. The unsteadiness of the problem leads to obtaining time-dependent results at any obliqueness angle.

\section{Problem formulation}

Axisymmetric incompressible oblique stagnation point flow impinges impulsively on a vertical circular cylinder with radius $a$ and the length of infinity with constant wall temperature, $T_{w}$, in the space influenced by gravity acceleration $g_{g}$. A schematic of this problem for two states of assisting and opposing flow against buoyancy forces is presented in Figure 1. The flow far from the cylinder surface can be considered inviscid which is composed of two perpendicular flows as: a normal stagnation flow onto the cylinder and a shear flow parallel to the cylinder axis. If the axisymmetric coordinate system $(r, z)$, according to Figure 1, is assumed on the cylinder in a way that always the separating streamline goes toward the positive $z$-direction, referring to Wideman et al. [6], then the oblique stream function and velocity components with coefficients $A>$ 0 and $B>0$ are:

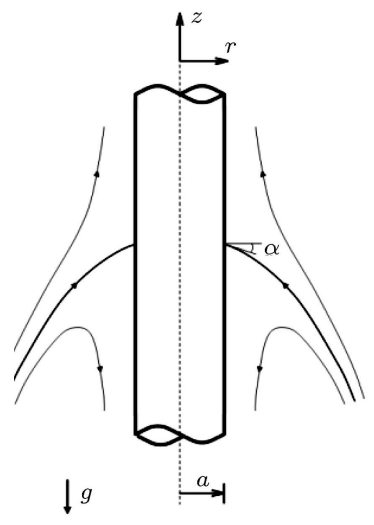

(a)

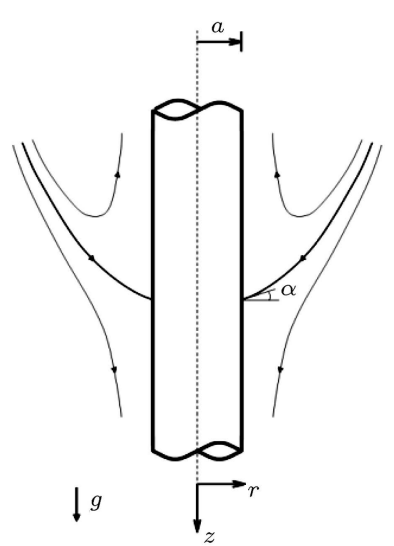

(b)
Figure 1. Schematic of the inviscid oblique stagnation flow impinging axisymmetrically on vertical circular cylinder at obliqueness angle $\alpha$ in the presence of buoyancy forces: (a) Assisting flow, and (b) opposing flow. 


$$
\begin{aligned}
& \psi_{\mathrm{inv}}=A\left(r^{2}-a^{2}\right) z+B \frac{1}{2 a}\left(r^{2}-a^{2}\right)^{2} \\
& \left\{\begin{array}{l}
U=-\frac{1}{r} \frac{\partial \psi}{\partial z}=-A\left(r-\frac{a^{2}}{r}\right) \\
W=\frac{1}{r} \frac{\partial \psi}{\partial r}=2 A z+2 B a\left(\frac{r^{2}}{a^{2}}-1\right)
\end{array}\right.
\end{aligned}
$$

It is easily seen that the velocity field of Eq. (2) obtained from the stream function expressed by Eq. (1) satisfies the continuity equation. In these relations, coefficients $A$ and $B$ indicate the strength of the normal stagnation flow and the axial shear flow, respectively. Their summation is equal to the total strength of oblique flow, $\bar{k}$, which is independent of the obliqueness angle. So:

$$
A+B=\text { Const. }=\bar{k} \text {. }
$$

\subsection{Flow obliqueness angle}

Since separating streamline $\psi_{\mathrm{inv}}=0$, derived from Eq. (1), is not a straight line and has a parabolic form, we use the tangent angle of this line at the incidence point on cylinder wall, named $\alpha$, as a scale of flow obliqueness. This definition, which is based on inviscid outer flow, is just used as a scale, and the correct tangent angle introduced by $\alpha_{s}$ will be calculated later which obviously has different values in practice due to fluid viscosity effects. To insert the influence of $\alpha$ into oblique flow equations, constants $A$ and $B$ in Eq. (1) should be determined. For $\psi_{\mathrm{inv}=0}$ in Eq. (1) under $r=a$ condition, we get:

$$
\tan \alpha=\left|\frac{d z}{d r}\right|_{\substack{\psi=0 \\ r=a}}=\frac{B}{A}
$$

Using Eqs. (3) and (4), we have:

$$
A=\frac{\cos \alpha}{\sin \alpha+\cos \alpha} \bar{k}, \quad B=\frac{\sin \alpha}{\sin \alpha+\cos \alpha} \bar{k} .
$$

\subsection{Boundary layer viscous flow}

To have flow field in the vicinity of cylinder surface (laminar boundary layer), we need to extract the governing equations in this region and solve them. These equations for incompressible flow in axisymmetric cylindrical coordinate system with velocity components $u(r, z, t)$ in $r$-direction, $w(r, z, t)$ in $z$-direction, and temperature field $T(r, z, t)$ are as follows:

Mass equation:

$$
\frac{\partial}{\partial r}(r u)+r \frac{\partial w}{\partial z}=0
$$

Momentum equations (Navier-Stokes equations):

$$
\begin{aligned}
\frac{\partial u}{\partial t} & +u \frac{\partial u}{\partial r}+w \frac{\partial u}{\partial z}=-\frac{1}{\rho} \frac{\partial p}{\partial r} \\
& +v\left(\frac{\partial^{2} u}{\partial r^{2}}+\frac{1}{r} \frac{\partial u}{\partial r}-\frac{u}{r^{2}}+\frac{\partial^{2} u}{\partial z^{2}}\right) \\
\frac{\partial w}{\partial t} & +u \frac{\partial w}{\partial r}+w \frac{\partial w}{\partial z}=-\frac{1}{\rho} \frac{\partial p}{\partial z} \\
& +v\left(\frac{\partial^{2} w}{\partial r^{2}}+\frac{1}{r} \frac{\partial w}{\partial r}+\frac{\partial^{2} w}{\partial z^{2}}\right) \pm g_{g} \beta\left(T-T_{\infty}\right)
\end{aligned}
$$

The last term in Eq. (8) is due to the presence of buoyancy forces in z-direction which is replaced by Boussinesq's approximation. Herein, $g_{g}$ is gravitational acceleration and $\beta$ is fluid volumetric thermal expansion coefficient. Positive sign is for the case of gravity acceleration in negative $z$-direction, so buoyancy forces are towards positive direction (flow direction), and we call it assisting flow case, whereas minus sign is for the case of positive $z$-direction gravity which makes buoyancy forces oppose the flow, and we call it opposing flow case (refer to Figure 1).

Energy equation:

$$
\frac{\partial T}{\partial t}+u \frac{\partial T}{\partial r}+w \frac{\partial T}{\partial z}=\alpha_{t h}\left(\frac{\partial^{2} T}{\partial r^{2}}+\frac{1}{r} \frac{\partial T}{\partial r}+\frac{\partial^{2} T}{\partial z^{2}}\right)
$$

where $\alpha_{t h}$ is thermal diffusivity. The boundary conditions of the problem with respect to the no-slip condition on the cylinder surface on one side and viscous flow conversion to inviscid flow far distant from the cylinder on the other side are:

$$
\begin{array}{ll}
r=a: & u=w=0 \text { and } T=T_{w}, \\
r \rightarrow \infty: & \left\{\begin{array}{l}
u=U \\
w=W
\end{array} \text { and } T=T_{\infty},\right.
\end{array}
$$

where $U$ and $W$ were already defined by Eq. (2). $T_{w}$ and $T_{\infty}$ are temperatures of cylinder wall and free steam flow, respectively. The stream function in viscous region $\left(\psi_{\text {vis }}\right)$ is considered as follows:

$$
\psi_{\text {vis }}=A a^{2} f(\eta) z+B a^{3} g(\eta) .
$$

So, we use similarity transformations for the velocity components (extracted from above equation) and temperature variable as in the following to transform the nonlinear momentum equations into the linear and solvable form, in which $A$ and $B$ are from Eq. (5):

$$
\left\{\begin{array} { l } 
{ u = - A a \eta ^ { - \frac { 1 } { 2 } } f ( \eta , \tau ) } \\
{ w = 2 A f ^ { \prime } ( \eta , \tau ) z + 2 B g ^ { \prime } ( \eta , \tau ) a } \\
{ \frac { T - T _ { \infty } } { T _ { w } - T _ { \infty } } = \theta ( \eta , \tau ) }
\end{array} \quad \left\{\begin{array}{l}
\eta=\left(\frac{r}{a}\right)^{2} \\
\tau=2 \bar{k} t
\end{array}\right.\right.
$$

These similarity variables satisfy continuity Eq. (6) 
automatically. By eliminating the pressure term from Eqs. (7) and (8), the following system of ODEs is yielded:

$$
\left\{\begin{array}{l}
\eta f^{\prime \prime \prime}+f^{\prime \prime}+\operatorname{Re}\left[m\left(1+f f^{\prime \prime}-f^{\prime 2}\right)-\frac{\partial f^{\prime}}{\partial \tau}\right]=0 \\
\eta g^{\prime \prime \prime}+g^{\prime \prime}+\operatorname{Re}\left[m\left(f g^{\prime \prime}-f^{\prime} g^{\prime}\right)-\frac{\partial g^{\prime}}{\partial \tau}\right]+\frac{\lambda}{n} \theta=0 \\
\eta \theta^{\prime \prime}+\theta^{\prime}+\operatorname{Re} \operatorname{Pr}\left(m f \theta^{\prime}-\frac{\partial \theta}{\partial \tau}\right)=0
\end{array}\right.
$$

Here, the prime sign denotes derivative with respect to $\eta$. Coefficients $m$ and $n$ along with Reynolds number, Prandtl number, and dimensionless parameter, $\lambda$, have the following definitions:

$$
\begin{aligned}
& m=\frac{\cos \alpha}{\sin \alpha+\cos \alpha}, \quad n=\frac{\sin \alpha}{\sin \alpha+\cos \alpha}, \\
& 0<\alpha<90^{\circ} \\
& \operatorname{Re}=\frac{a^{2} \bar{k}}{2 v}, \quad \operatorname{Pr}=\frac{v}{\alpha_{t h}}, \quad \lambda= \pm \frac{g_{g} \beta a\left(T_{w}-T_{\infty}\right)}{8 v \bar{k}} .
\end{aligned}
$$

Also, the following boundary conditions for the ODE system (13) are deduced by considering Eq. (10):

$$
\left\{\begin{array}{lll}
f(1, \tau)=0, & f^{\prime}(1, \tau)=0, & f^{\prime}(\infty, \tau)=1 \\
g(1, \tau)=0, & g^{\prime}(1, \tau)=0, & g^{\prime \prime}(\infty, \tau)=1 \\
\theta(1, \tau)=1, & \theta(\infty, \tau)=0 &
\end{array}\right.
$$

Since the unsteadiness of the problem is a consequence of the impulsive motion of the free stream flow at $t=0$, we consider the fluid at rest (no motion) with uniform temperature, $T_{\infty}$, in both fluid and cylinder's wall for $t<0$ as the initial conditions. For $t \geq 0$, a sudden free stream flow, according to Eq. (2), appears, and therefore, the cylinder's wall temperature rises to $T_{w}$.

It is notable here that due to the presence of dimensionless axial velocity function, $g$, the effect of buoyancy forces is included by $(\lambda / n) \theta$, unlike that presented by Gorla [11] in which buoyancy term $(\operatorname{Re} \xi) \theta$ was inserted into $f$-equation, and a linearly-varying wall temperature against $z$ was assumed in order to obtain the self-similar form of the equations.

\subsection{Flow characteristics}

Now, we are able to calculate some flow characteristics using the resulting data of functions $f, g$, and $\theta$. Nusselt number and shear-stress on the cylinder wall are obtained by the following relations:

$$
\begin{aligned}
& \mathrm{Nu}=\frac{h a}{2 k}=\frac{-k\left[\frac{\partial T}{\partial r}\right]_{r=a} \times a}{2 k\left(T_{w}-T_{\infty}\right)}=-\theta^{\prime}(1, \tau), \\
& \tau_{w}=\mu\left(\frac{\partial w}{\partial r}\right)_{r=a}=4 \mu \bar{k}\left[\frac{m z}{a} f^{\prime \prime}(1, \tau)+n g^{\prime \prime}(1, \tau)\right] .
\end{aligned}
$$

The position of incidence point of the separating streamline on cylinder surface, $z_{s}$, where the shear stress is zero, and the slope of this streamline at incident point, $\operatorname{tg} \alpha_{s}$, are calculated by:

$$
\begin{aligned}
z_{s}=\left.z\right|_{\tau_{w}=0}=-a \tan \alpha \frac{g^{\prime \prime}(1, \tau)}{f^{\prime \prime}(1, \tau)}, \\
\operatorname{tg} \alpha_{s}=\left[\left(\frac{d z}{d r}\right)_{\psi_{\text {vis }}=0}\right]_{r=a} \\
=-\frac{2}{3} \operatorname{tg} \alpha\left[\frac{m \operatorname{Re} g^{\prime \prime}(1, \tau)+\frac{\lambda}{n} f^{\prime \prime}(1, \tau)}{f^{\prime \prime 2}(1, \tau)}\right] .
\end{aligned}
$$

\section{Method of solution}

Eqs. (13) along with boundary conditions (15) are solved numerically using an implicit finite-difference scheme. The third-order differential equations for $f$ and $g$ are reduced to second-order equations by substituting $f^{\prime}=F$ and $g^{\prime}=G$. Then, all firstorder and second-order derivatives with respect to $\eta$ are discretized by using a central difference formulation, whereas the first-order derivatives in $\tau$ are replaced by a backward difference approximation.

The nonlinear terms are converted to linear form by using a Taylor series expansion about $\tau+\Delta \tau$. The solution procedure starts at $\tau=\Delta \tau$ as the first timestep with respect to the known values at $\tau=0$ as initial conditions to determine the unknown values of $F, G$, and $\theta$ functions by using the well-known Thomas algorithm. The procedure is iterated until the results converge with the stable values by an appropriate accuracy. The same procedure is repeated for the next time-step and the problem solution continues to a value of $\tau$, where the results show a steady-state behavior. We used $\Delta \eta=0.01, \Delta \tau=0.001$, and $\eta_{\max }=50$ as an upper limit for calculation domain to reach accuracy up to fourth decimal places. The latter value is an appropriate choice because it would be observed that boundary layer thickness in the worst case does not exceed $\eta=10$. We employed several $\Delta \eta$ to have a best choice of grid size along $\eta$. For $\Delta \eta=0.01$ to $\Delta \eta=0.1$, the results are exactly the same, and hence they are independent of grid size; for values less than 0.01 , due to accumulation of truncation errors, no convergence with our expected accuracy would occur.

\section{Results and discussions}

The results presented here are produced by solving ODEs system (13) along with boundary conditions (15) for certain values $\operatorname{Re}=1, \operatorname{Pr}=0.7$, and $\lambda=1$ in the range of obliqueness angle $\alpha$ between 0 and $80^{\circ}$.

Distribution of radial velocity function, $f^{\prime}(\eta, \tau)$, for $\alpha=30^{\circ}$, which is obtained at three-time levels $\tau=0.01,0.1,0.5$, as well as the steady-state condition, 
which is illustrated by dashed line, is shown in Figure 2. Distribution of thermal function, $\theta(\eta, \tau)$, is depicted in Figure 3 for $\alpha=30^{\circ}$ and the same time levels. According to these diagrams, by increasing obliqueness angle $\alpha$, the domain of variation of $f^{\prime}$ and $\theta$ functions (the boundary layer is influenced by them) increases which is due to the reduction of the normal flow strength.

Distributions of axial velocity function $g^{\prime}(\eta, \tau)$ for $\alpha=30^{\circ}, \operatorname{Re}=1, \operatorname{Pr}=0.7$, and $\lambda=1$ and the same time levels in the two cases of assisting and opposing flows are presented in Figure 4. The initial convexity of the curves in Figure 4(a) is due to the buoyancy forces which act in the direction of the shear flow parallel to the cylinder axis. Due to the positive effect of the buoyancy forces on the assisting flow, the convexity is

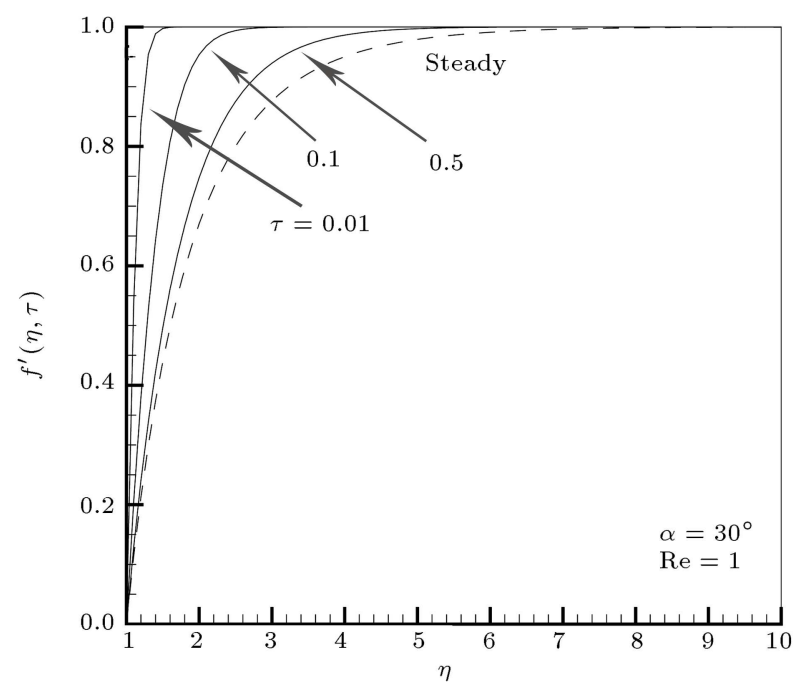

Figure 2. Distribution of the radial velocity function, $f^{\prime}(\eta, \tau)$, for $\operatorname{Re}=1$ and $\alpha=30^{\circ}$ at different time levels $\tau=0.01,0.1,0.5, \infty$.

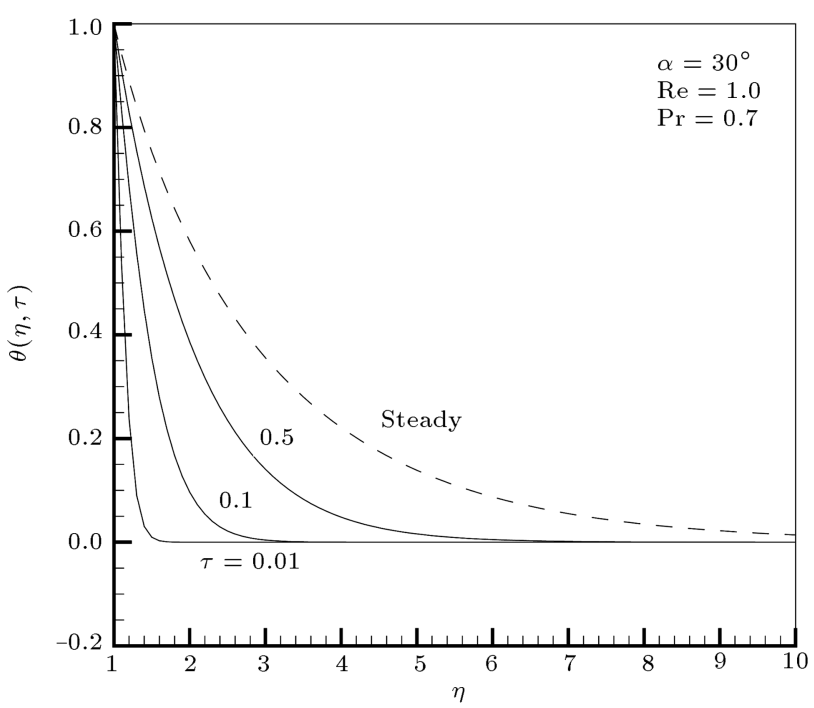

Figure 3. Distribution of the thermal function $\theta(\eta, \tau)$ for $\operatorname{Re}=1, \operatorname{Pr}=0.7$, and $\alpha=30^{\circ}$ at different time levels, $\tau=0.01,0.1,0.5, \infty$.

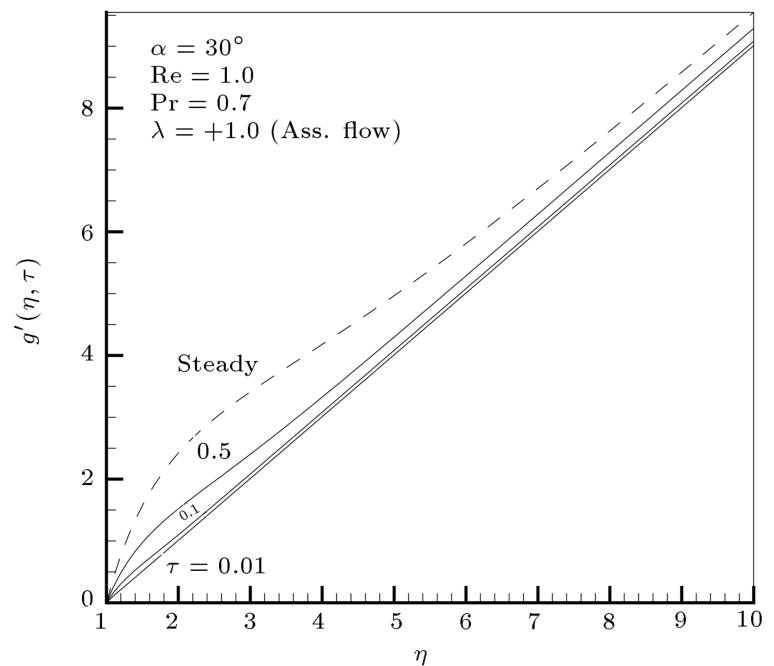

(a)

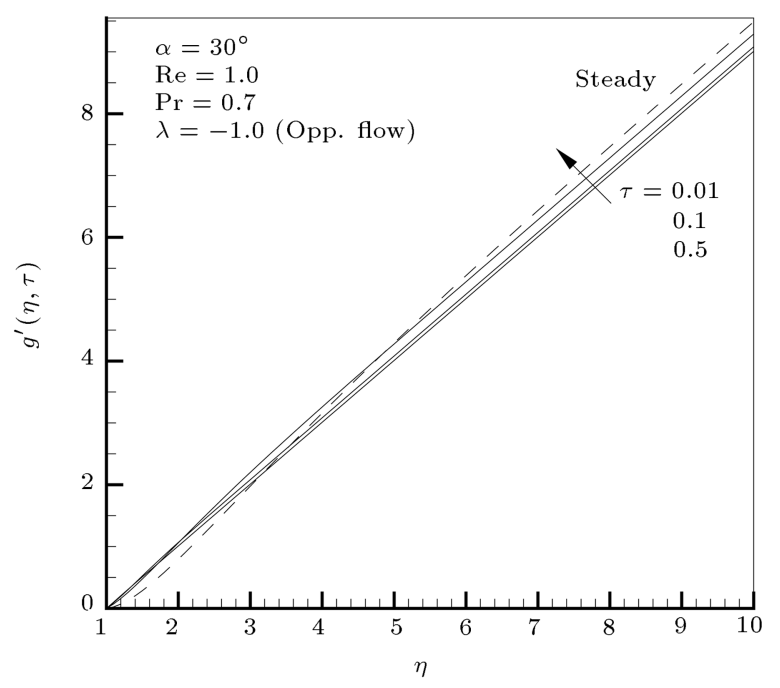

(b)

Figure 4. Distribution of the axial velocity function $g^{\prime}(\eta, \tau)$ for $\operatorname{Re}=1, \operatorname{Pr}=0.7$, and $\alpha=30^{\circ}$ at different time parameters $\tau=0.01,0.1,0.5, \infty$ : (a) Assisting flow; and (b) opposing flow.

toward positive side too, but in the case of the opposing flow, it is downward (toward negative $z$ ). As it can be seen, for initial time levels, there is an insignificant convexity, especially in the assisting flow case which is due to the buoyancy forces not extended yet.

The presented diagrams in Figure 5 are related to the variation of boundary layer thickness based on the distribution of functions $f^{\prime}(\eta)$ and $\theta(\eta)$ drawn for $\alpha=10^{\circ}, 30^{\circ}, 60^{\circ}$. $\eta_{99 \%}$ is defined as a value of $\eta$ where the value of the function reaches $99 \%$ of its target. This diagram indicates the time level where the steady-state condition is reached for velocity and/or thermal distributions. According to these diagrams, for obliqueness angle, $\alpha=10^{\circ}$, we have a steady condition of velocity $f^{\prime}$ at about $\tau=2$, whereas this situation for the temperature distribution starts from 


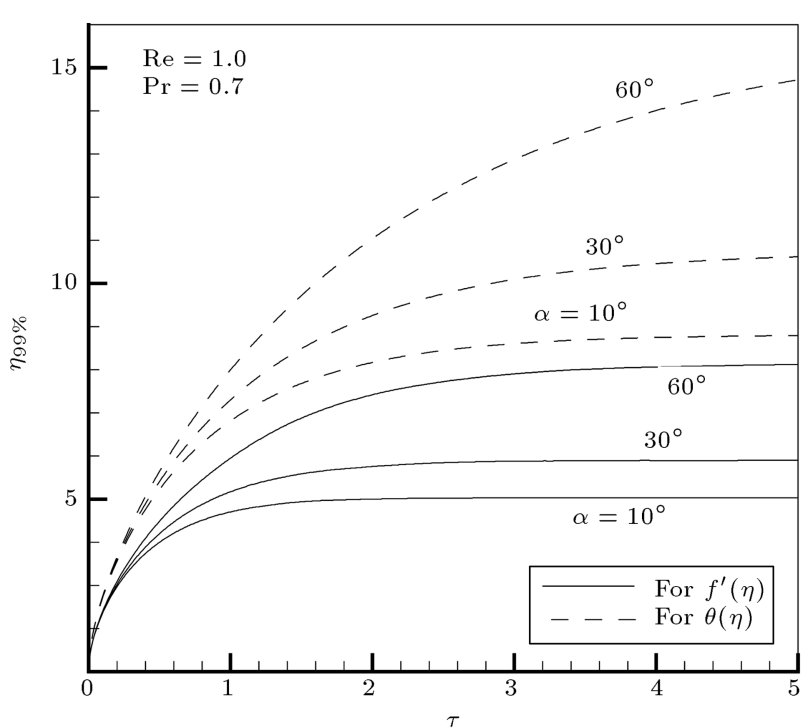

Figure 5. Variation of boundary layer thickness, $\eta_{99 \%}$, based on distribution of $f^{\prime}$ and $\theta$ for constant values of $\operatorname{Re}=1, \operatorname{Pr}=0.7$ and different obliqueness angles, $\alpha=10^{\circ}, 30^{\circ}, 60^{\circ}$.

$\tau=4.5$. Besides, for larger obliqueness angles, we witness a delay in the starting time of the steadystate condition. Another point in the diagrams is the existence of a larger thermal boundary layer in comparison to dynamic boundary layer at any angle $\alpha$.

The variation of boundary value, $f^{\prime \prime}(1, \tau)$, against obliqueness angle for $\mathrm{Re}=1$ at different time levels, $\tau=0.01,0.02,0.1,0.5$, is observed in Figure 6 . Also, the same variation for $\theta^{\prime}(1, \tau)$, which is equal to Nusselt number multiplied by a minus sign, is shown in Figure 7 . According to these diagrams, both $f^{\prime \prime}(1, \tau)$ function and Nusselt number decrease very slightly against $\alpha$, but as time passes, the slope of this reduction grows and it continues to the steady-state condition when the rate of the reduction in curves is the highest. For better illustration, a comparison of Nusselt number variation against $\alpha$ in different time instants is presented in Table 1 . Here, we see that for the initial moments, e.g. $\tau=0.01$, Nusselt number varies from 5.133 to 5.139 when $\alpha$ changes from zero to $50^{\circ}$. It means that at this instant of time, $50^{\circ}$ variation of the flow obliqueness angle makes only $0.08 \%$ reduction in Nusselt number. But, at the next time levels, $\tau=0.02,0.1,0.5$, the steady-state Nusselt number would reduce with more percent like $0.16,0.85$, 4.45, and 23.6.

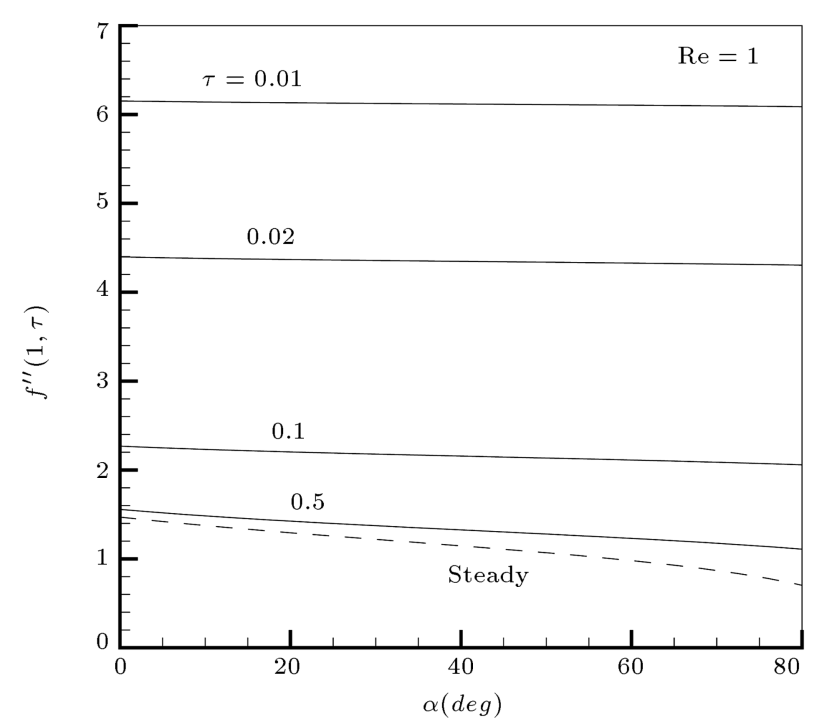

Figure 6. Variation of the boundary value, $f^{\prime \prime}(1, \tau)$, against obliqueness angle, $\alpha$, for $\operatorname{Re}=1$ at different time parameters, $\tau=0.01,0.02,0.1,0.5, \infty$.

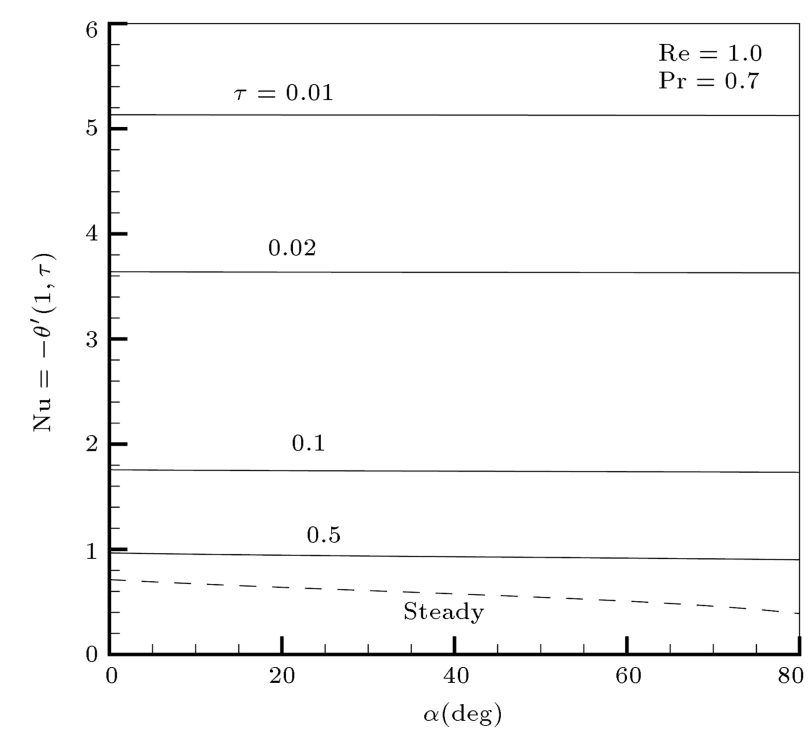

Figure 7. Variation of Nusselt number (boundary value $\left.\theta^{\prime}(1, \tau)\right)$ against the obliqueness angle, $\alpha$, for $\operatorname{Re}=1$ and $\operatorname{Pr}=0.7$ at different time parameters, $\tau=0.01,0.02,0.1$, $0.5, \infty$.

The variations of boundary value, $g^{\prime \prime}(1, \tau)$, against the obliqueness angle for $\operatorname{Re}=1, \operatorname{Pr}=0.7$, and $\lambda=1$ at different time levels, $\tau=0.01,0.02,0.1,0.5$, in the both states of assisting and opposing flows are shown

Table 1. Comparison of Nusselt number variation against obliqueness angle, $\alpha$, in the different time instants for Re $=1$ and $\operatorname{Pr}=0.7$.

\begin{tabular}{|c|c|c|c|c|c|c|c|c|c|c|}
\hline & \multicolumn{2}{|c|}{$\tau=0.01$} & \multicolumn{2}{|c|}{$\tau=0.02$} & \multicolumn{2}{|r|}{$\tau=0.1$} & \multicolumn{2}{|r|}{$\tau=0.5$} & \multicolumn{2}{|c|}{ Steady state } \\
\hline & $\mathbf{N u}$ & $\begin{array}{c}\text { Variation } \\
\text { percentage }\end{array}$ & $\mathrm{Nu}$ & $\begin{array}{c}\text { Variation } \\
\text { percentage }\end{array}$ & $\mathrm{Nu}$ & $\begin{array}{c}\text { Variation } \\
\text { percentage }\end{array}$ & $\mathrm{Nu}$ & $\begin{array}{c}\text { Variation } \\
\text { percentage }\end{array}$ & $\mathrm{Nu}$ & $\begin{array}{c}\text { Variation } \\
\text { percentage }\end{array}$ \\
\hline $\begin{array}{l}\alpha=0^{\circ} \\
\alpha=50^{\circ}\end{array}$ & $\begin{array}{l}5.133 \\
5.129\end{array}$ & $-0.08 \%$ & $\begin{array}{l}3.639 \\
3.633\end{array}$ & $-0.16 \%$ & $\begin{array}{l}1.756 \\
1.741\end{array}$ & $-0.85 \%$ & $\begin{array}{l}0.966 \\
0.923\end{array}$ & $-4.45 \%$ & $\begin{array}{l}0.712 \\
0.544\end{array}$ & $-23.6 \%$ \\
\hline
\end{tabular}


in Figure 8. According to the diagrams, the value of $g^{\prime \prime}(1, \tau)$ function on the both sides of $\alpha$ 's range faces a high growth, specifically in the steady-state condition. For the assisting flow case, at the initial time levels, no growth is observed in the diagrams for large values of $\alpha$; however, in steady-state condition, we see a growing behavior. From diagram of Figure $8(\mathrm{~b})$, it is notable that $g^{\prime \prime}(1, \tau)$ changes sign from negative to positive at a specific obliqueness angle in all time levels. Besides, it is interesting to note that all the diagrams, regardless of their time levels, meet at the same value at obliqueness angle of about $50^{\circ}$.

The variation of boundary value, $f^{\prime \prime}(1, \tau)$, against dimensionless time, $\tau$, for three different angles $\alpha=$

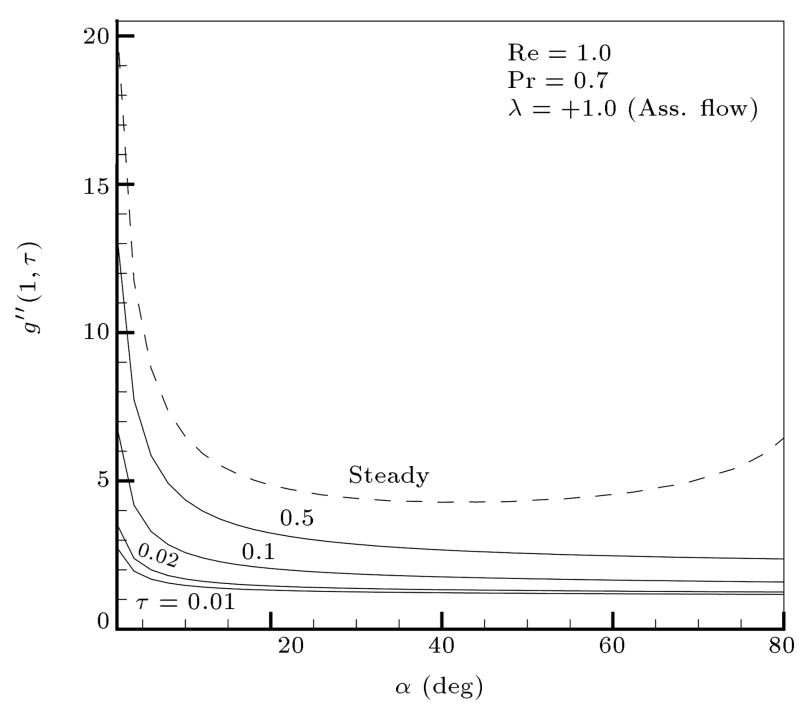

(a)

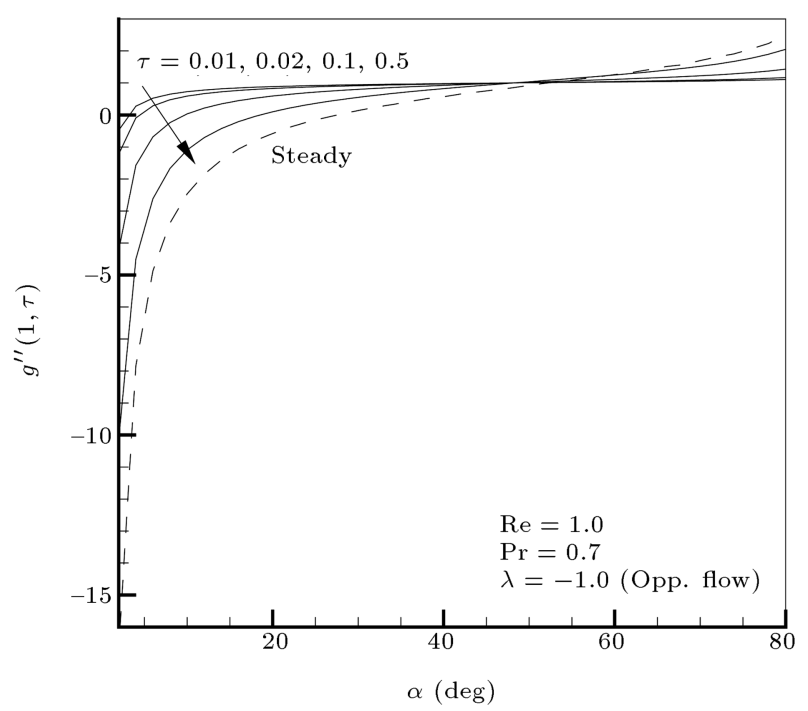

(b)

Figure 8. Variation of boundary value, $g^{\prime \prime}(1, \tau)$, against obliqueness angle, $\alpha$, for $\operatorname{Re}=1$ and $\operatorname{Pr}=0.7$ at different time parameters, $\tau=0.01,0.02,0.1,0.5, \infty$ : (a) Assisting flow, and (b) opposing flow. $10^{\circ}, 30^{\circ}, 60^{\circ}$ is presented until $\tau=1$ in Figure 9. Similarly, the variation of Nusselt number is drawn until $\tau=3$ in Figure 10. The obvious fact in these two figures is the drastic fall of curves at a short period of initial time at all different obliqueness angles which happens for $f^{\prime \prime}(1, \tau)$ at about $\tau<0.05$ and for Nusselt number at about $\tau<0.4$ (Nusselt number varies from 5.1 to about 1 at this time interval). From then on, the functions vary more moderately (Nusselt number varies from 1 to steady value 0.6 ) and they divert from each other for different values of $\alpha$. In other words, at higher time instants, specifically at steady-state, effect of the obliqueness angle on Nusselt number would be more sensible.

Moreover, the variations of boundary value, $g^{\prime \prime}(1, \tau)$, against dimensionless time, $\tau$, for $\operatorname{Re}=1$,

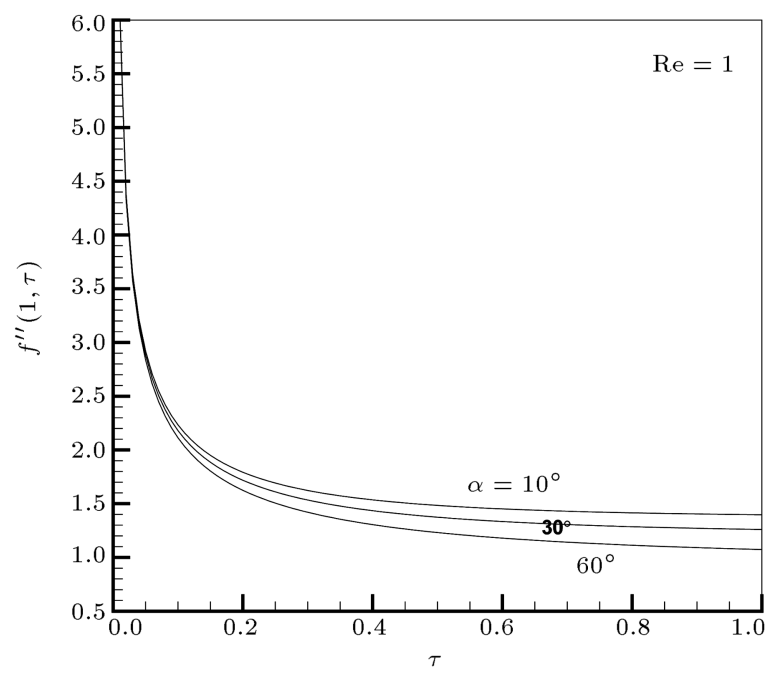

Figure 9. Variation of boundary value, $f^{\prime \prime}(1, \tau)$, against time for $\operatorname{Re}=1$ and different obliqueness angles, $\alpha=10^{\circ}$, $30^{\circ}, 60^{\circ}$.

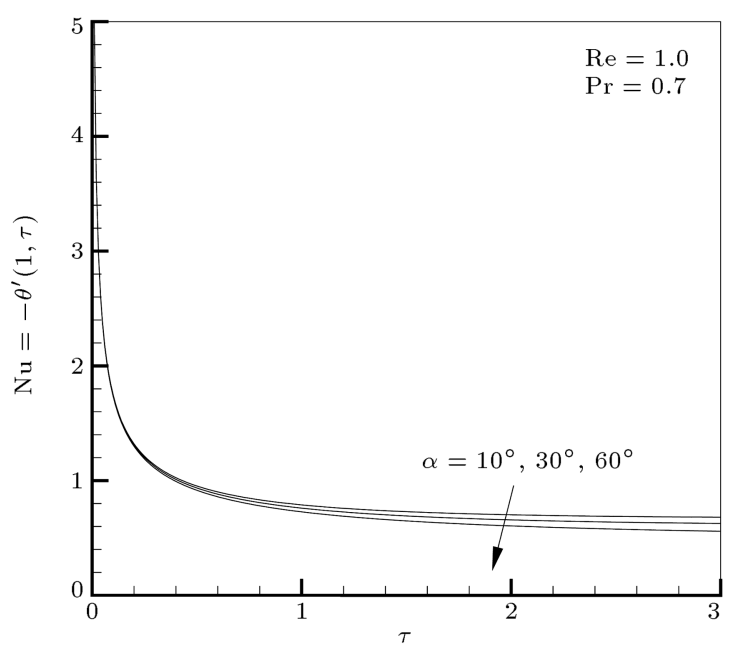

Figure 10. Variation of Nusselt number (boundary value $\left.\theta^{\prime}(1, \tau)\right)$ against the time for $\operatorname{Re}=1, \operatorname{Pr}=0.7$ and different obliqueness angles, $\alpha=10^{\circ}, 30^{\circ}, 60^{\circ}$. 
$\operatorname{Pr}=0.7$, and $\lambda=1$ and three different angles $\alpha=10^{\circ}$, $30^{\circ}, 60^{\circ}$ are represented until $\tau=5$ (Figure 11), which is distinguished for two cases of the assisting and opposing flows. According to Figure 11(a) (assisting flow), $g^{\prime \prime}(1, \tau)$ is always positive and grows as time passes for any value of $\alpha$. However, its variation for $\alpha=30^{\circ}$ takes a substantial drop compared to that for $\alpha=10^{\circ}$, and in $\alpha=60^{\circ}$, it has a variation close to $\alpha=30^{\circ}$, so that the two diagrams can meet each other at about $\tau=5$. In the case of the opposing flow (Figure 11(b)), the variation of $g^{\prime \prime}(1, \tau)$ shows different behaviors in various angles, $\alpha$. It has a descending trend against $\tau$ for $\alpha=10^{\circ}$ and $30^{\circ}$, but an ascending trend for $\alpha=60^{\circ}$. This event is due to the effects of the opposing buoyancy forces too, which are negligible versus the axial flow when $\alpha$ becomes high.

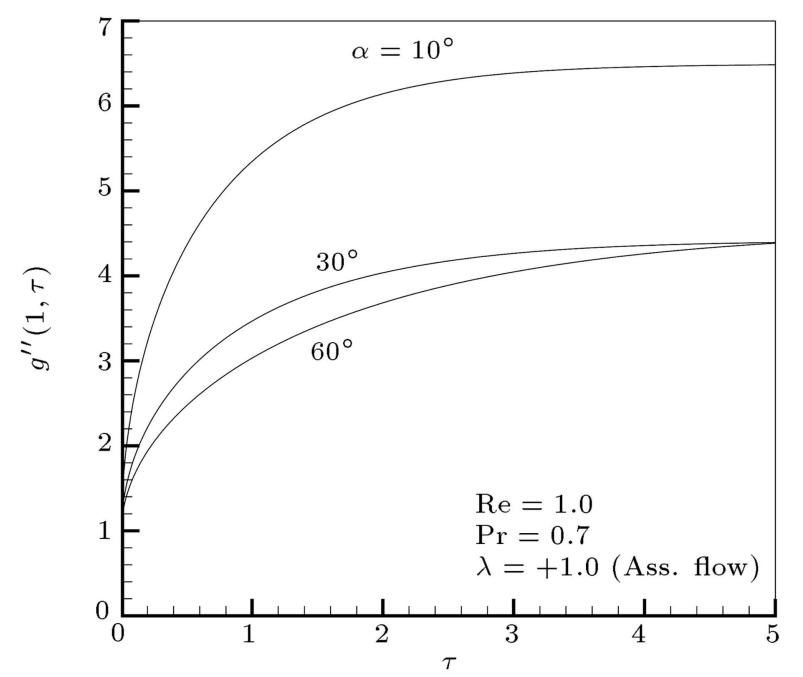

(a)

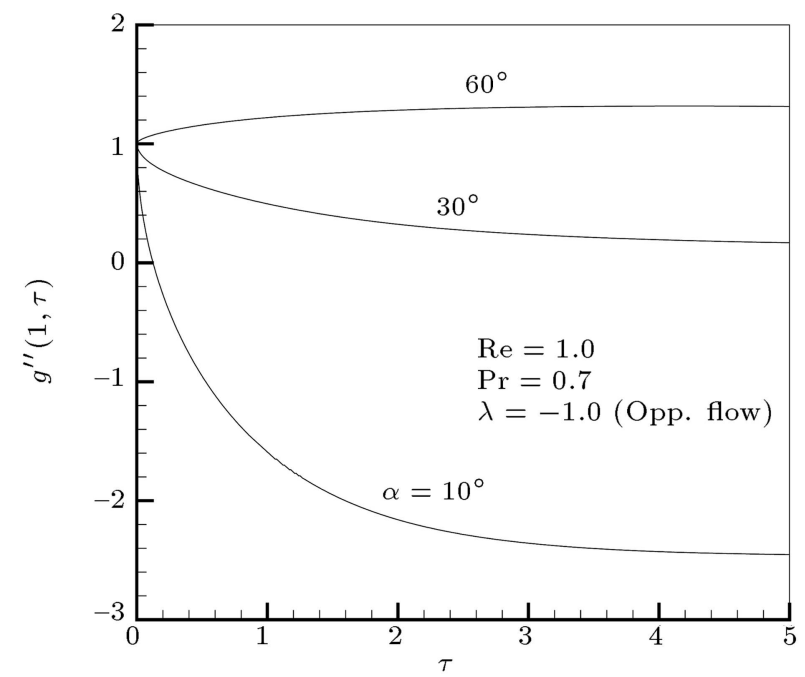

(b)

Figure 11. Variation of boundary value, $g^{\prime \prime}(1, \tau)$, against the time for $\operatorname{Re}=1, \operatorname{Pr}=0.7$ and different obliqueness angles, $\alpha=10^{\circ}, 30^{\circ}, 60^{\circ}$ : (a) Assisting flow, and (b) opposing flow.

\section{Conclusions}

Effect of obliqueness angle of the flow in unsteady problem of mixed convection heat transfer from a vertical cylinder impinged by an impulsive axisymmetric oblique stagnation-point flow on the characteristics of boundary layer zone has been investigated. For the outer flow which is supposed to be inviscid, simple analytical equations govern, but in the boundary layer region near the cylinder surface, nonlinear equations of viscous flow have been reduced to a system of semi-similar equations solved by using finite-difference method. Considering constant strength for the outer flow in the specific case of $\operatorname{Re}=1, \operatorname{Pr}=0.7$, and $\lambda=1$, the solution process has been repeated for the selected arbitrary angles of obliqueness, from zero (net normal stagnation flow) to about $90^{\circ}$ (net axial flow) as the buoyancy forces have assisting or opposing effects. The numerical results of the problem demonstrate variation of different parameters such as Nusselt number or boundary value of the dimensionless axial and radial velocity functions, $f^{\prime \prime}(1, \tau)$ and $g^{\prime \prime}(1, \tau)$, against the time and flow obliqueness angle, $\alpha$. According to the resulting diagrams, by increasing the angle of obliqueness, $\alpha$, boundary value $f^{\prime \prime}(1, \tau)$ and Nusselt number decrease slightly, whereas boundary value $g^{\prime \prime}(1, \tau)$ has an intense growth on both sides of $\alpha$ 's range. Also, through increasing time, $f^{\prime \prime}(1, \tau)$ and Nusselt number show a moderate reduction to steady-state condition after a sudden drop at the initial instants. At the beginning of the flow, Nusselt number is high, e.g. about 5.1 at $\tau=0.01$, also approximately independent of $\alpha$ 's value. After a short period of time (for $\tau>0.4$ ), Nusselt number begins to get close to a certain steady value corresponding to its related obliqueness angle $\alpha$, so that in the steady-state condition, which occurs for $\tau>3$, we can reach $\mathrm{Nu}=0.67,0.61,0.51$ for $\alpha=10^{\circ}$, $30^{\circ}, 60^{\circ}$, respectively. From numerical point of view, we observe that in $\tau=0.01$, Nusselt number equals 5.133 and 5.139 for $\alpha=0$ and $\alpha=50^{\circ}$, respectively, which shows only $0.08 \%$ variation, whereas, in the steady-state condition, the values of Nusselt number for the same values of $\alpha$ reach 0.712 and 0.544 , which means a $23.6 \%$ reduction.

\section{Nomenclature}

$a$

A

Radius of cylinder

$B$

Strength of radial flow

Strength of shear flow

$f, g \quad$ Dimensionless velocity functions

$g_{g} \quad$ Gravitational acceleration

$h \quad$ Convective heat transfer coefficient

$k \quad$ Thermal conductivity

$\bar{k} \quad$ Strength of free stream flow 


\begin{tabular}{|c|c|}
\hline$m, n$ & Coefficients \\
\hline $\mathrm{Nu}$ & Nusselt number \\
\hline $\operatorname{Pr}$ & Prandtl number \\
\hline$p$ & Dynamic pressure \\
\hline $\operatorname{Re}$ & Reynolds number \\
\hline$r$ & Radial direction of cylinder \\
\hline$r^{*}$ & Dimensionless $r,(r / a)$ \\
\hline$t$ & Time \\
\hline$T$ & Temperature \\
\hline$T_{w}$ & Cylinder wall temperature \\
\hline$T_{\infty}$ & Free stream temperature \\
\hline$u, w$ & $\begin{array}{l}r \text { - and } z \text {-component of velocity of } \\
\text { viscous flow }\end{array}$ \\
\hline$U, W$ & $\begin{array}{l}r \text { - and } z \text {-component velocity of inviscid } \\
\text { flow }\end{array}$ \\
\hline$z$ & Cylinder axial direction \\
\hline$z_{s}$ & $z$-value of incident point \\
\hline$\alpha$ & Obliqueness angle \\
\hline$\alpha_{s}$ & Tangent angle at incident point \\
\hline$\alpha_{t h}$ & Thermal diffusivity \\
\hline$\beta$ & $\begin{array}{l}\text { Volumetric thermal expansion } \\
\text { coefficient }\end{array}$ \\
\hline$\eta$ & Dimensionless parameter \\
\hline$\lambda$ & $\begin{array}{l}\text { Dimensionless mixed convection } \\
\text { parameter }\end{array}$ \\
\hline$\theta$ & Dimensionless temperature function \\
\hline$\mu$ & Dynamic viscosity \\
\hline$\nu$ & Kinematics viscosity \\
\hline$\rho$ & Density \\
\hline$\tau$ & Dimensionless $t,(2 \bar{k} t)$ \\
\hline$\tau_{w}$ & Shear-stress on cylinder wall \\
\hline$\psi_{\text {inv }}$ & Inviscid flow stream function \\
\hline$\psi_{\text {vis }}$ & Viscous flow stream function \\
\hline
\end{tabular}

\section{References}

1. Hiemenz, K. "Die Grenzchicht an einem in den gleichformingen Flussigkeitsstrom eingetauchten graden Kreiszylinder", Dinglers Polytech. J., 236, pp. 321-410 (1911).

2. Wang, C. "Axisymmetric stagnation flow on a cylinder", Quaterly of Applied Mathematics, 10, pp. 207213 (1974).

3. Gorla, R.S.R. "Heat transfer in axisymmetric stagnation flow on a cylinder", Applied Scientific Research J., 32, pp. 541-553 (November, 1976).

4. Gorla, R.S.R. "Transient response behavior of an axisymmetric stagnation flow on a circular cylinder due to time dependent free stream velocity", Int. $J$. Engineering Science, 16, pp. 493-502 (1978).
5. Gorla, R.S.R. "Unsteady viscous flow in the vicinity of an axisymmetric stagnation point on a circular cylinder", Int. J. Engineering Science, 17, pp. 87-93 (1979).

6. Takhar, H.S., Chamkha, A.J. and Nath, G. "Unsteady axisymmetric stagnation-point flow of a viscous fluid on a cylinder", Int. Journal of Engineering Science, 37, pp. 1943-1957 (1999).

7. Stuart, J.T. "The viscous flow near a stagnation point when the external flow has uniform vorticity", Journal of the Aerospace Sciences, 26(2), pp. 124-125 (1959).

8. Okamoto, H. "Axisymmetric stagnation flow converging obliquely on a cylinder", J. Phys. Soc. Japan, 64(8), pp. 2714-2717 (1995).

9. Weidman, P.D. and Putkaradze, V. "Axisymmetric stagnation flow obliquely impinging on a circular cylinder", European Journal of Engineering B. Fluids, 22, pp. 121-131 (2003).

10. Ramachandran, R., Chen, T.S. and Armaly, B.F. "Mixed convection in stagnation flows adjacent to vertical surfaces", ASME J. Heat Transf, 110, pp. 373377 (1988).

11. Gorla, R.S.R. "Mixed convection in an axisymmetric stagnation flow on a vertical cylinder", Acta Mech., 99, pp. 113-123 (1993).

12. Lok, Y.Y., Amin, N.I. and Pop, I. "Mixed convection near a non-orthogonal stagnation- point flow on a vertical plate with uniform surface heat flux", Acta Mech., 186, pp. 99-112 (2006).

13. Revnic, C., Grosan, T., Merkin, J.H. and Pop, I. "Mixed convection flow near an axisymmetric stagnation-point on a vertical cylinder", J. Eng. Math., 64(1), pp. 2909-2913 (2009).

\section{Biographies}

Reza Bayat was born in Mashhad, Iran, in 1977. He received his BS degree in Mechanical Engineering from Ferdowsi University of Mashhad in 1999 and his MS degree in Mechanical Engineering from Sharif University of Technology in Mechanical Engineering in 2001. He is currently a PhD candidate in Ferdowsi University of Mashhad.

Asghar Baradaran Rahimi was born in Mashhad, Iran, in 1951. He received his BS degree in Mechanical Engineering from Tehran polytechnic, in 1974, and a $\mathrm{PhD}$ degree in Mechanical Engineering from the University of Akron, Ohio, U.S.A., in 1986. He has been a Professor in the Department of Mechanical Engineering at Ferdowsi University of Mashhad since 2001. His research and teaching interests include heat transfer and fluid dynamics, gas dynamics, continuum mechanics, applied mathematics, and singular perturbations. 